\title{
La torre nella torre. Recupero e rilievo 3D per la fruizione della Torre Matta ad Otranto
}

The tower in the tower. Recovery and 3D survey for virtual visits to the Torre Matta in Otranto

\author{
Giovanna Muscatello \\ Dipartimento di Beni Culturali - Università del Salento, Lecce, Italy, giovannamuscatello@libero.it
}

\begin{abstract}
Otranto is one of the biggest ancient settlements in the Salento (Puglia), in the easternmost part of the Italian peninsula. This location has always affected the city's history, which has a stratified system of defence. As a result of the Turkish invasion of 1480, the city was completely destroyed. During the counter-offensive of 1481 the city was reconquered by the Aragonese, who are credited with the reconstruction of the city and its defences, building high walls with circular towers (still visible and well conserved), which housed artillery pieces on the various floors of the casemates. Around the mid sixteenth century the existing defensive structures were enriched with bastions including the imposing pentagonal structure that incorporates the circular tower of the late fifteenth century, the so-called Torre Matta, facing the harbour. As part of recent recovery measures, the enormous room inside the bastion was completely emptied. This entailed removing all the accumulated material which, over the years, had come to fill the entire space. This material obscured the external wall of the fifteenth century tower enclosed within the bastion, of which, at the beginning of the work, only the stone corbels and the blind arches at the top were visible. The material had also prevented access via the only original entrance, on the southeast side, which was on the level of the moat. The stratified deposits to be removed were about $18 \mathrm{~m}$ deep, and the operation served to bring to light the entire room and the tower, making it possible for the first time to appreciate the relationship between the walls. To record the geometry of the individual architectural features, a 3D laser scan was performed, integrated with direct surveys. A three-dimensional model was created in order to enable virtual visits and disseminate knowledge of the monument.
\end{abstract}

Keywords: Otranto, Torre Matta, laser scanner 3D, virtual tour.

\section{Introduzione}

Il presente studio è stato condotto nell'ambito dell'intervento di valorizzazione di uno dei più singolari monumenti della città di Otranto, la Torre Matta (Figg.1 e 2).

Le attività hanno previsto il recupero della torre, svuotandola dal materiale di accumulo presente all'interno fino alla base, il restauro delle super- fici murarie con interventi reversibili e la riqualificazione delle aree esterne alla quota dei fossati.

Considerate le notevoli dimensioni del vano, con l'intradosso della volta posto a circa $17 \mathrm{~m}$ dal piano di calpestio, il rilievo tridimensionale ha documentato per la prima volta la singolare conformazione dell'edificio che al suo interno cu- 
stodisce parte di una rondella quattrocentesca (Fig. 3). Dal rilievo laser scanner 3D è stato possibile estrapolare un tour virtuale progettato nella nuvola di punti che costituisce un documento unico per la conservazione digitale dello stato di fatto prima dell'installazione delle strutture previste dal progetto (Fig. 4).

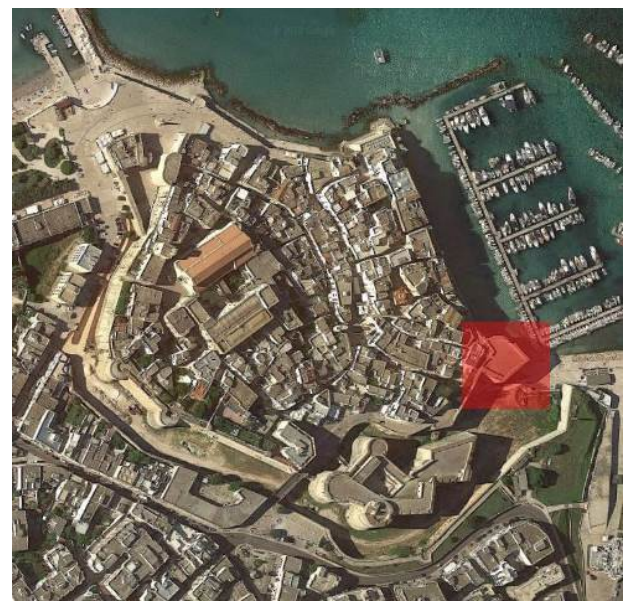

Fig. 1. Otranto. In rosso è evidenziata la posizione della Torre Matta rispetto al nucleo storico della città (Earth Google).

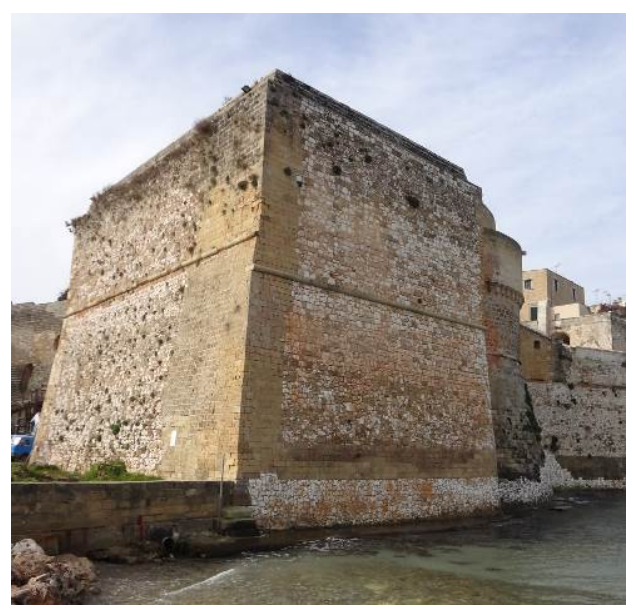

Fig. 2. Otranto. Il Bastione della Torre Matta visto dall'area portuale.

\section{Inquadramento storico-archeologico}

Otranto è uno dei maggiori insediamenti antichi del Salento nel punto più orientale della penisola italiana (I.G.M. 215 III NO); questa ubicazione ha da sempre condizionato la storia della città $\mathrm{e}$ del territorio circostante nonché il ruolo politico e sociale di questo centro. Il territorio ha restituito testimonianze che attestano una frequentazione antropica lungo un considerevole arco cronologico che si snoda dalla preistoria sino ad oggi. Otranto in età tardo-antica, conquistato il ruolo predominante nei traffici marittimi del canale, diventa la destinazione finale delle Vie Appia e Traiana. Nel periodo medievale era una città portuale a metà strada tra Costantinopoli, capitale dell'Impero d'Oriente, e Roma, centro dell'Impero d'Occidente. L'invasione turca, nel 1480 , è stato l'episodio più cruento della storia della città durante il quale il centro venne completamente distrutto con ripercussioni gravissime su tutto il territorio salentino. La controffensiva si ebbe nel 1481 quando la città venne riconquistata dagli Aragonesi a cui seguì una graduale ricostruzione ed una ripresa con un conseguente incremento demografico. Agli aragonesi si deve la riorganizzazione urbana e difensiva della città, con l'edificazione di alte mura a scarpa, torri circolari e la ridefinizione del fossato. Verso la fine del XV secolo il sistema difensivo della città venne ampliato e alle torri esistenti furono aggiunte: la Torre della Duchesca e la Rondella, posta sul mare all'estremità del Bastione dei Pelasgi, poi incorporata parzialmente dal bastione conosciuto oggi come Torre Matta.

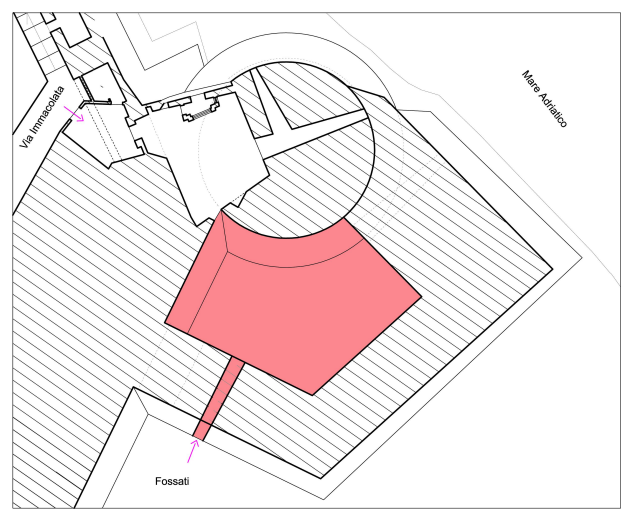

Fig. 3. Torre Matta. Planimetria. In evidenza il vano interno svuotato e rilevato con laser scanner 3D. 


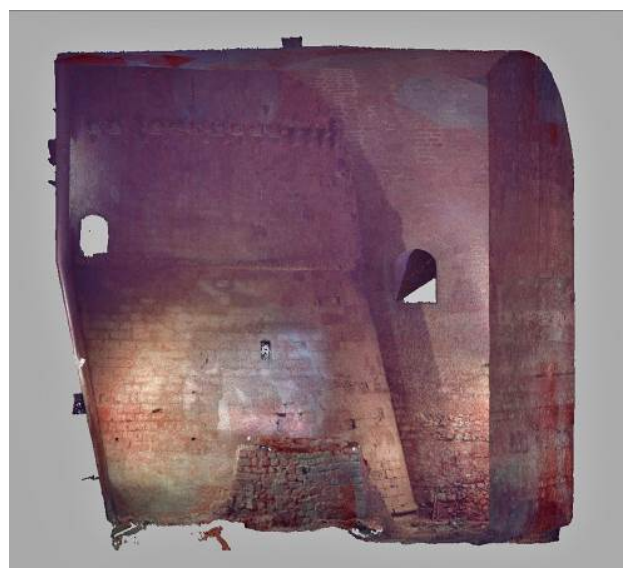

Fig. 4. Torre Matta. Rilievo laser scanner 3D del vano interno al bastione che ingloba parte della rondella quattrocentesca (Mitello \& Muscatello, 2014).

\section{La Torre Matta: lo svuotamento del vano e l'analisi del complesso monumentale}

Il bastione pentagonale è ubicato a ridosso dell'area portuale ed è stato edificato intorno alla metà del XVI secolo per potenziare le strutture difensive esistenti nel settore settentrionale della città di Otranto, a salvaguardia delle cortine esterne maggiormente esposte al tiro e all'attacco assediante. Il bastione ingloba parte della rondella edificata alla fine del XV secolo. L'enorme vano presente all'interno del bastione è stato interamente liberato dal materiale di accumulo, riversato all'interno nel corso degli anni, che aveva occultato l'intero ambiente e il paramento esterno della rondella quattrocentesca di cui erano visibili, all'inizio delle lavorazioni, solo i beccatelli lapidei e gli archetti ciechi posti in alto (Fig. 5). L'accesso originario al bastione, posto a sud-ovest alla quota dei fossati, era impraticabile, occluso da pietre e terra compattati (Fig. 6). Prima dello svuotamento l'accesso al vano del bastione era possibile solo da una sala collocata all'interno della rondella quattrocentesca posta ad un livello superiore e raggiungibile dal centro storico (Fig. 7).

Da qui hanno avuto inizio le operazioni di svuotamento che si sono rivelate molto complesse e delicate poiché la rimozione dei depositi stratificati ha interessato un'altezza di circa $13 \mathrm{~m}$.

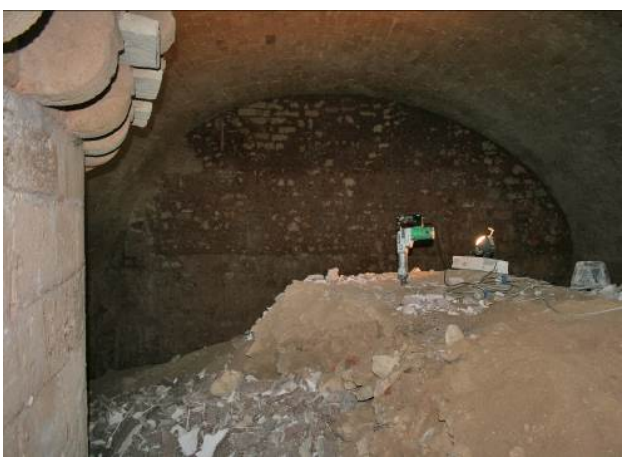

Fig. 5. L'interno del bastione prima dello svuotamento.

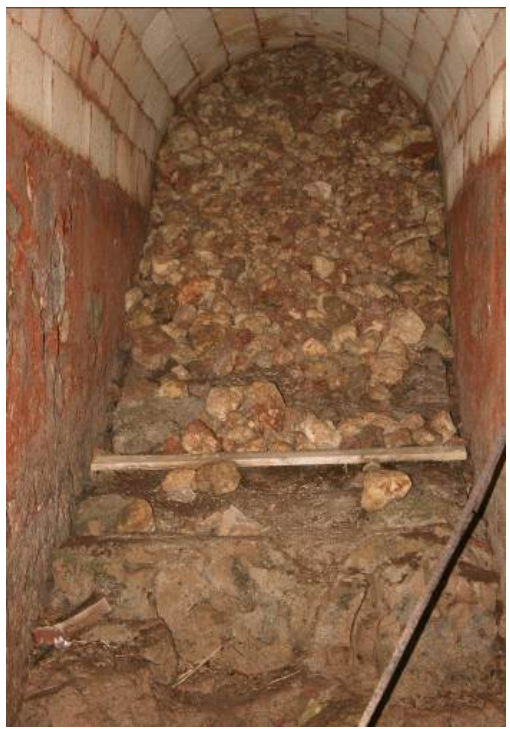

Fig. 6. Ingresso originario alla torre alla quota dei fossati prima della rimozione del materiale di accumulo.

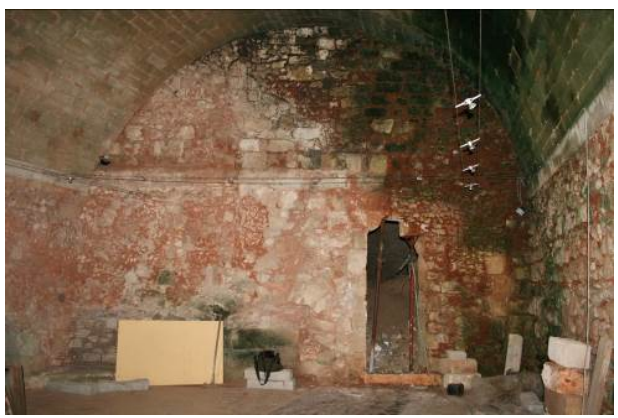

Fig. 7. Sala al piano superiore. Il varco nel muro, sulla destra, era l'unico accesso alla Torre Matta prima dello svuotamento. 
Inoltre l'assenza di coerenza e la scarsa stabilità degli accumuli non garantiva una tecnica valida per ogni settore del vano, dato che si ignorava la natura dei materiali sepolti. Il materiale rimosso era di vario tipo: scarti edili, detriti, terra, blocchi in cemento relativi a demolizioni recenti di abitazioni del centro storico, mentre nella parte più bassa un cumulo di macerie proveniente da svariati contesti limitrofi in cui sono stati individuati e recuperati blocchi lapidei modanati unitamente a frammenti ceramici moderni, rinascimentali e medievali; per decenni questo vano è stato utilizzato come luogo di discarica. I lavori sono stati condotti con un regolare e continuativo controllo archeologico che ha consentito di verificare preventivamente la presenza di evidenze archeologiche o il possibile reimpiego di blocchi messapici o medievali nella costruzione del paramento murario aragonese. Contestualmente è stata effettuata la pulitura del paramento murario del bastione, della rondella quattrocentesca e della parete a scarpa aragonese, le quali si sono rivelate in un ottimo stato di conservazione non essendo esposte ad agenti esogeni. La messa in luce delle cortine murarie e la scoperta degli elementi distintivi delle strutture ha chiarito aspetti costruttivi relativi alle differenti fasi storiche. Lungo il prospetto ovest, sono state rilevate la traccia di un archibugiera a foro tondo e la merlatura che coronava la parte sommitale delle mura prima dell'intervento di sopraelevazione della fine del XV secolo ad opera degli aragonesi (Fig. 8).

Alle spalle della cortina probabilmente si articola il camminamento esterno di ronda, oggi non più praticabile poiché sono state addossate abitazioni moderne. Inoltre, lungo il prospetto ovest sono stati rinvenuti pezzi di nucleo della cortina aragonese con una malta costituita da terra rossa, inerti in pietra calcarea e bauxite macinata.

Sono stati messi in evidenza anche gli elementi caratteristici della rondella quattrocentesca tra cui una bocca di fuoco, che sostituì probabilmente l'archibugiera della fase precedente, e le prese d'aria o sfiatatoi, essenziali per liberare i camminamenti dai fumi nocivi delle polveri delle artiglierie. Si tratta di elementi funzionali necessari nelle operazioni belliche e distribuiti all'interno della torre nei vari livelli.

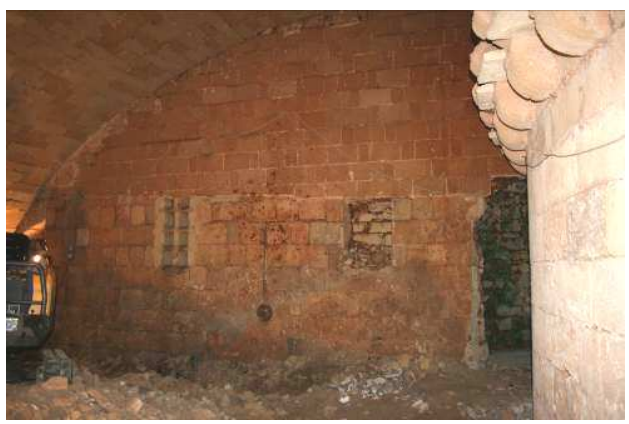

Fig. 8. Cortina muraria posta ad ovest. In evidenza l'archibugiera a foro tondo con lunga fessura verticale e la traccia della merlatura che coronava la struttura difensiva prima di essere sopraelevata alla fine del XV secolo.

\subsection{Il ritrovamento della struttura muraria}

Durante le ultime fasi di svuotamento del vano interno al puntone, a circa $4 \mathrm{~m}$ dall'ingresso originario alla base, è emersa una struttura muraria di non chiara funzione, forse un'opera di cantiere mai rimossa (Figg. 9 e 10). La struttura muraria poggiava su un terreno di riporto e si addossava alla torre circolare quattrocentesca.

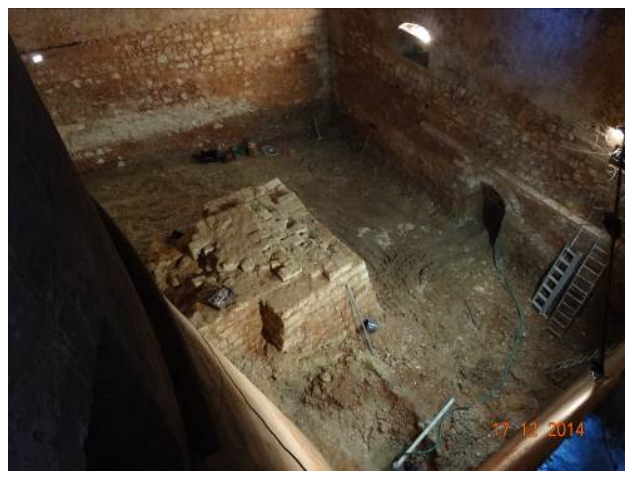

Fig. 9. Struttura muraria scoperta alla base del vano.

Presentava una conformazione architettonica insolita: una faccia frontale con profilo a scarpa e pareti laterali rastremate verso la rondella a cui si appoggiava con un misto di conci e terra. La struttura è stata edificata con elementi costitutivi disomogenei; i blocchi presentavano dimensioni variabili, alcuni di riutilizzo e provenienti da contesti storici diversi. 
Da un punto di vista planimetrico era costituita da due elementi: un primo corpo murario di forma rettangolare che si addossava alla rondella ed un rivestimento a forma di " $U$ " che inglobava parzialmente il primo nucleo. Tale rivestimento è pertinente ad una seconda fase costruttiva a sostegno della struttura esistente per sopperire all'assenza di una fondazione stabile.

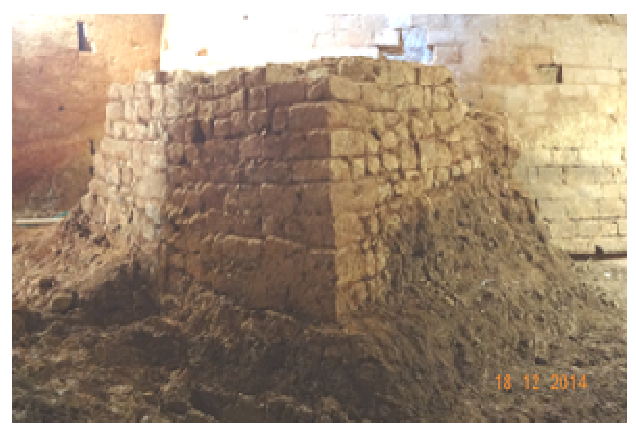

Fig. 10. Struttura muraria addossata alla rondella.

La presenza nella muratura di elementi di riutilizzo come un blocco lapideo sagomato, plausibilmente il concio di un arco, di un segmento del toro marcapiano appartenente alla rondella quattrocentesca, e di alcuni frammenti ceramici dello stesso periodo, colloca l'edificazione di questa struttura muraria dopo il XVI secolo, quando la rondella fu trasformata ed inglobata nel puntone.

A seguito dell'autorizzazione rilasciata dalla Soprintendenza Archeologica della Puglia la struttura muraria emersa durante le operazioni di scavo è stata rimossa. Lo smontaggio è stato effettuato per strati orizzontali e rispettando i piani di allettamento dei blocchi. Durante lo smontaggio dei blocchi è stata verificata immediatamente la condizione di precarietà della struttura. Infatti, la tecnica costruttiva e le caratteristiche meccaniche dei materiali impiegati, composti principalmente da blocchi sbozzati, pietrame di varia pezzatura, ciottoli ed alcuni elementi di reimpiego non hanno conferito particolare stabilità alla costruzione. Si può ipotizzare ad un'opera accessoria di cantiere, mai tolta e di difficile datazione. Sicuramente successiva alla rondella quattrocentesca, visto il riutilizzo di un segmento del toro marcapiano. La parte retrostante della struttura muraria era poggiata su un terrapieno argil- loso poco compatto ed intriso d'acqua. Un'ulteriore aspetto che viene chiarito una volta tolto il materiale all'interno del bastione è la forma costruttiva della rondella che è stata eretta rispettando le caratteristiche orografiche della roccia degradante verso il mare. Infatti, il basamento della torre e il toro marcapiano si fondono con il banco roccioso; quest' ultimo viene sagomato creando un solido zoccolo con profilo lievemente a scarpa che si armonizza con il resto della costruzione. Sfruttare la roccia per la fondazione ha conferito maggiore solidità alla struttura difensiva (Fig. 11).

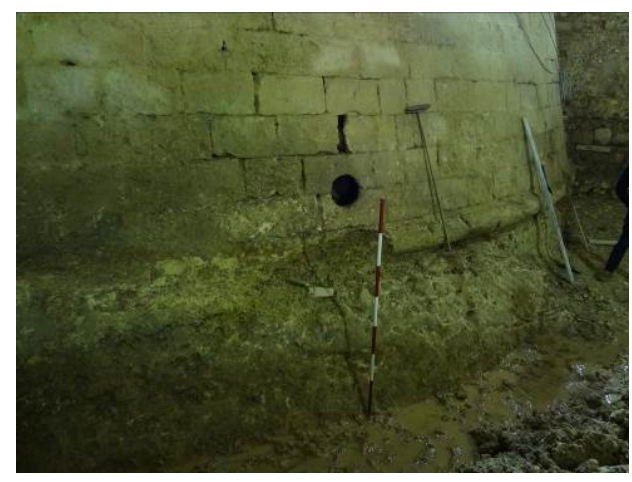

Fig. 11. Rondella quattrocentesca. In evidenzia il basamento con il toro marcapiano che si innestano nel banco roccioso di fondazione.

\subsection{Il rilievo diretto e il rilievo tridimensiona- le}

Dopo gli interventi di svuotamento il bastione si mostra come un maestoso ambiente alto circa 17 $\mathrm{m}$, sormontato da una volta a botte con imposte oblique senza piani intermedi e dotato di cannoniere sia a nord che a sud. A nord ingloba parte della torre circolare del Bastione dei Pelasgi, dal basamento troncoconico con toro marcapiano alla base, mentre ad ovest si appoggia alla cortina muraria aragonese con profilo a scarpa e costruita a filari isometrici. Sul paramento murario interno est, costituito da pietrame di varia pezzatura misto a terra e bolo, sono ancora ben visibili i piani di attesa distinguibili dalle diverse cromie e la finitura realizzata con intonaco con inclusi di bauxite. Un opera ben apparecchiata che presuppone la presenza di maestranze molto qualificate che hanno reperito il materiale da costru- 
zione nella zona circostante. Prima di provvedere allo smontaggio della posticcia struttura muraria, resosi necessario vista la precarietà del piano fondale, sono stati effettuati un'attenta analisi storico-architettonica ed un rilievo metrico diretto e strumentale al fine di comprendere gli aspetti dimensionali e le caratteristiche tecniche del manufatto in relazione allintero complesso architettonico e per valutare i rapporti tra le varie unità stratigrafiche murarie (Figg. 12-14).

Inoltre, considerata la singolarità del monumento esaminato e a completamento della registrazione delle particolarità dell'opera architettonica che possiamo definire come "la Torre nella Torre", è stato realizzato un rilievo laser scanner 3D finalizzato non solo alla registrazione delle informazioni metriche e colorimetriche ma soprattutto all'analisi e all'apprezzamento della struttura nella sua totalità, documentando la tecnica muraria usata in paramento, i materiali le modifiche strutturali e individuando le opere realizzate dagli aragonesi e i rapporti temporali tra le strutture (Fig. 15).

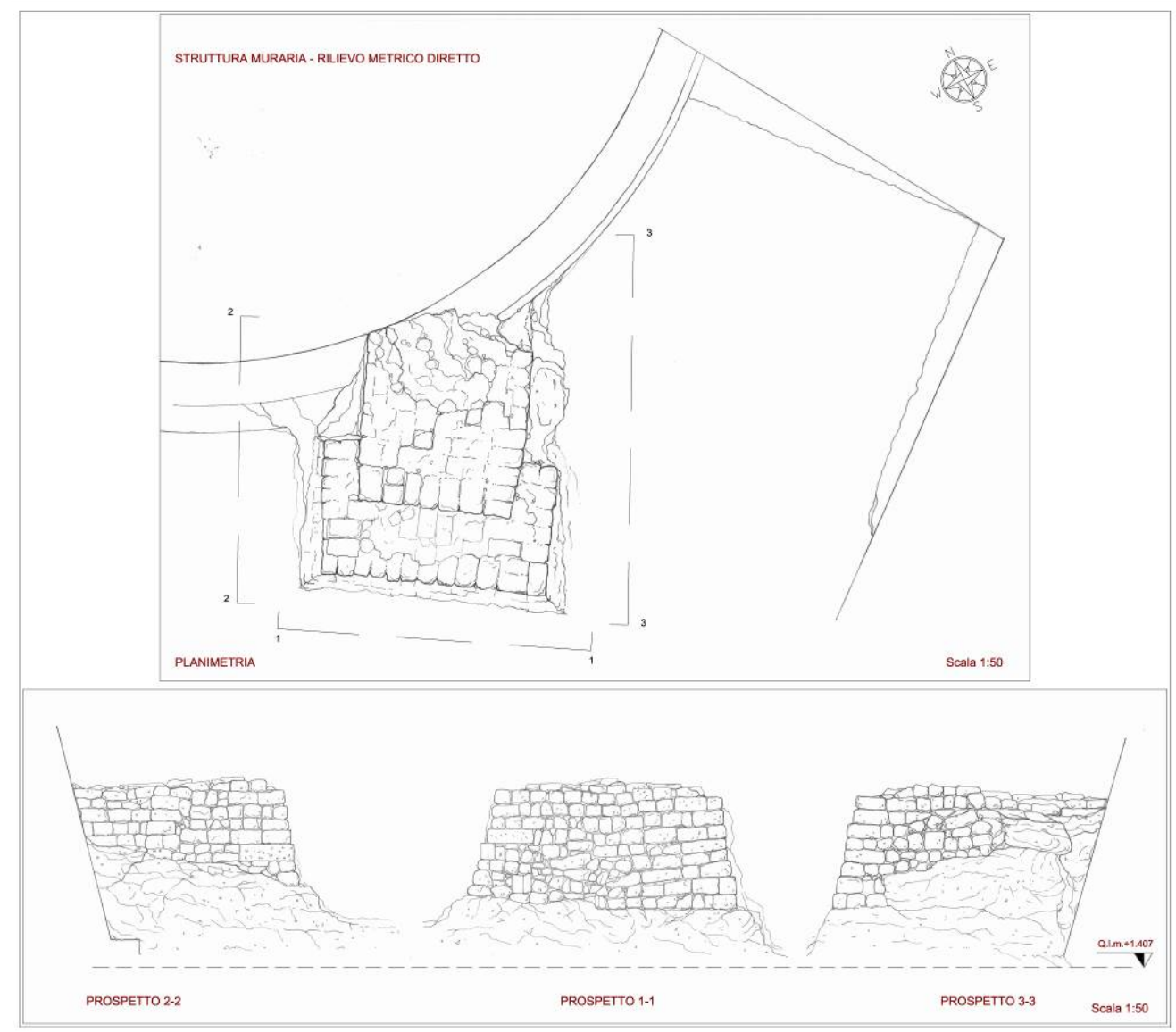

Fig. 12. Rilievo metrico diretto della struttura muraria addossata alla rondella quattrocentesca con caratterizzazione degli elementi costitutivi (elaborazione grafica G. Muscatello, 2014). 


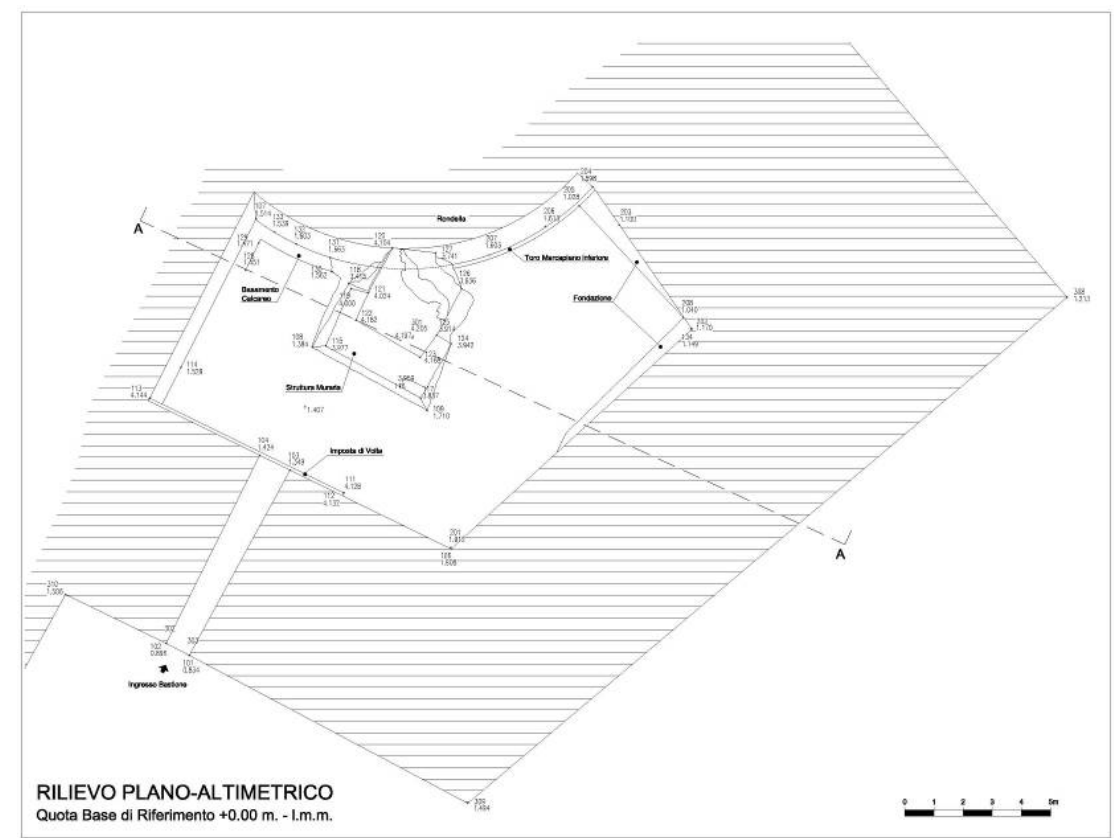

Fig. 13. Torre Matta. Rilievo plano-altimetrico strumentale del bastione, della rondella quattrocentesca e della struttura muraria messa in luce durante le operazioni di scavo. In evidenza il lungo corridoio di accesso al vano posto alla quota dei fossati (Mitello \& Muscatello, 2014).

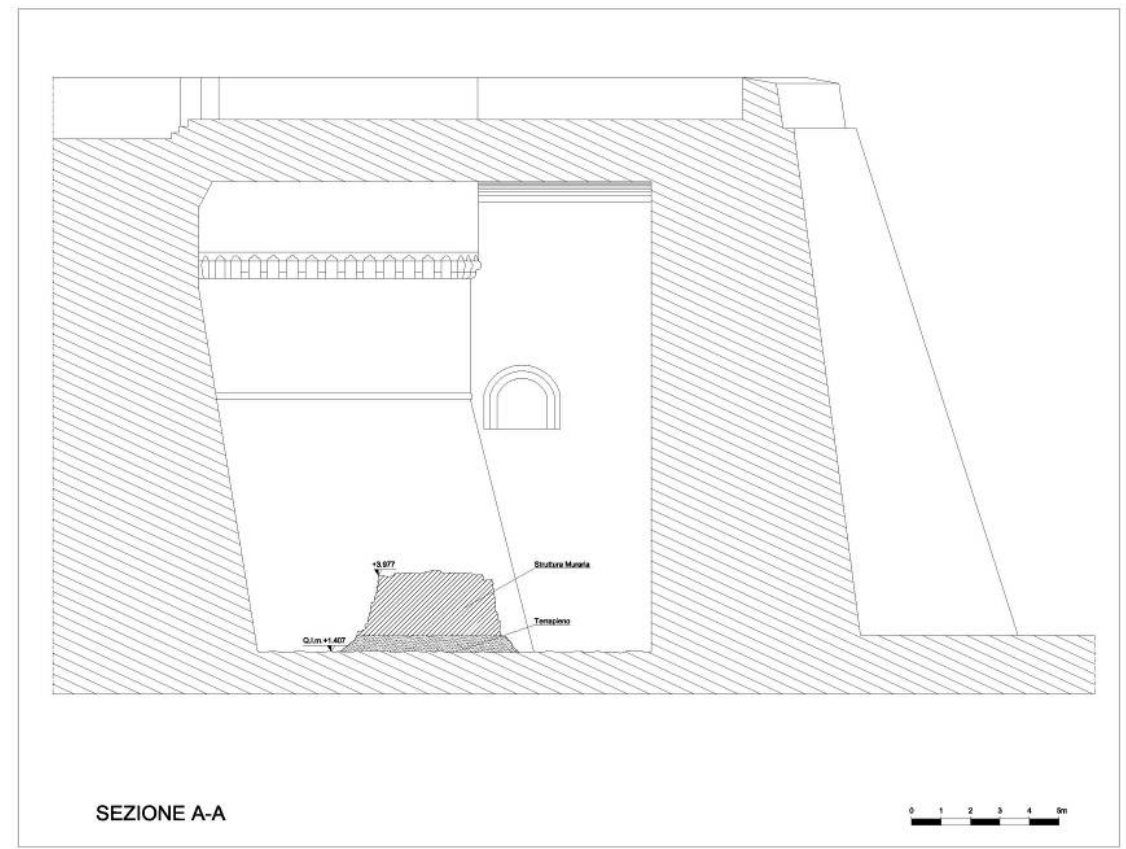

Fig. 14. Rilievo metrico strumentale della Torre Matta. Nella sezione prospettica si evidenzia il rapporto tra il bastione, la torre circolare e la struttura muraria (Mitello \& Muscatello, 2014). 

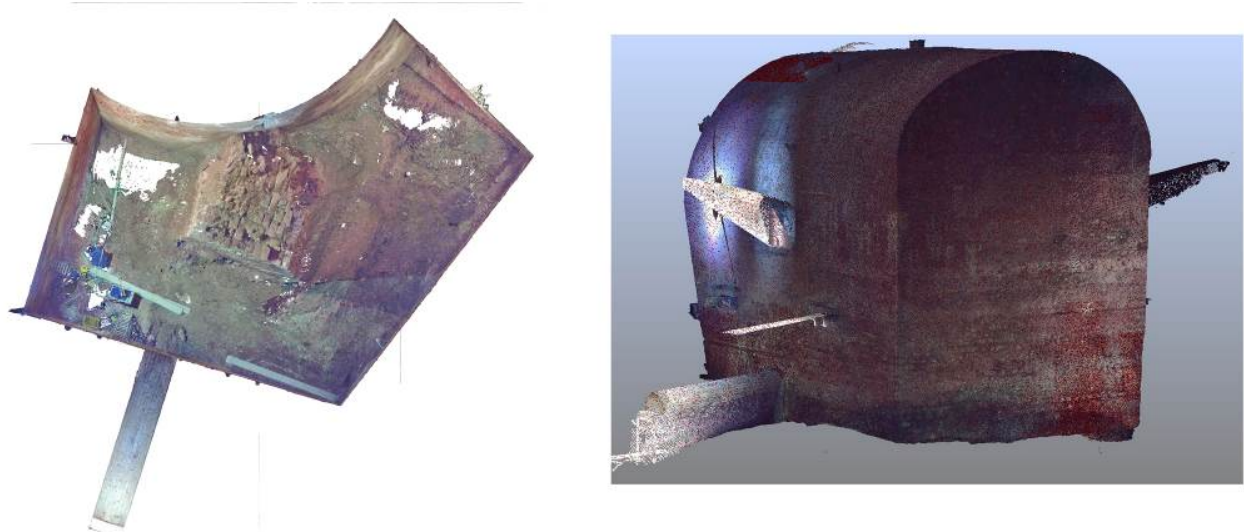

Fig. 15. A sinistra, ortofoto da rilievo laser scanner 3D del vano interno al bastione. A destra, modello 3D da nuvola di punti del volume interno. In evidenza la conformazione plano-volumetrica (Mitello \& Muscatello, 2014).

\section{Conclusioni}

Obiettivo precipuo del presente studio è quello di far conoscere la Torre Matta con tutte le sue peculiarità costruttive. Grazie ai lavori di recupero è stato possibile restituire alla comunità questo singolare monumento che, come una capsula del tempo, ha conservato una parte della storia del sistema difensivo della città. La realizzazione di un tour virtuale all'interno del modello tridimensionale scaturito dal rilievo laser scanner 3D, eseguito nella fase finale dello svuotamento del vano, permette una fruizione digitale integrale delle componenti geometriche e strutturali dell'organismo architettonico attraverso una nuvola di punti che nel contempo rimane un database di quanto ritrovato.

\section{Note}

La ricerca è stata condotta all'interno delle attività di sorveglianza archeologica previste durante le operazioni svuotamento del bastione. Otranto, Torre Matta - Lavori di Interventi per la Ristrutturazione, il Recupero e la Fruizione della Torre Matta ad Otranto. Intervento finanziato con i fondi POIn "Attrattori culturali, naturali e turismo" PO FESR PUGLIA 2007/2013 Asse I, Linea d'intervento I.1.1.

I rilievi diretti e strumentali e le elaborazioni grafiche sono stati realizzati da Giovanna Muscatello e Carmine Mitello e rimangono di proprietà degli autori.

\section{Bibliography}

Angelini, G.; Carlone, G. (1985). Castelli e fortificazioni in Puglia, visite alle difese marittime nell'età del viceregno spagnolo, Cavallino Ed.

Cariddi, P. (2014). Otranto intra moenia, dagli Aragonesi ad oggi, Esperidi Ed., Monteroni di Lecce.

Muscatello, G.; Quarta, A.; Mitello, C. (2018). "I Sotterranei dei castelli di Otranto e Gallipoli: dal rilievo laser scanner 3D all'analisi strutturale", in Marotta, A.; Spallone, R., eds., Defensive Architecture of the Mediterranean, Politecnico di Torino Ed., Torino, vol. 9, pp. 1021-1028.

Vita, R. de. (1984). Castelli, torri ed opere fortificate di Puglia, Adda Editore, Bari. 